\title{
Correction to: HIV-1 genetic diversity, geographical linkages and antiretroviral drug resistance among individuals from Pakistan
}

\author{
Saeed Khan ${ }^{1} \cdot$ Maria Zahid $^{2} \cdot$ Muhammad Asif Qureshi $^{3}$. \\ Nouman Mughal $^{3} \cdot$ Ikram Din Ujjan $^{4}$
}

Published online: 19 October 2017

(C) Springer-Verlag GmbH Austria 2017

\section{Correction to: Arch Virol \\ DOI 10.1007/s00705-017-3564-1}

Unfortunately, the co-author's name Nouman Mughal was incorrectly published in the original article and the same is corrected here in this erratum.

The online version of the original article can be found under doi:10.1007/s00705-017-3564-1.

Saeed Khan

saeed.khan@duhs.edu.pk

1 Department of Pathology (Molecular Pathology), Dow International Medical College, Dow University of Health Sciences, Karachi, Pakistan

2 Dow Diagnostic, Research \& Reference Laboratories (Molecular Pathology), Dow University of Health Sciences, Karachi, Pakistan

3 Department of Pathology, Dow International Medical College, Dow University of Health Sciences, Karachi, Pakistan

4 Department of Pathology, Liaquat University of Medical and Health Sciences, Jamshoro, Pakistan 